A - Research concept and design

B - Collection and/or assembly of data

$\mathrm{C}$ - Data analysis and interpretation

D - Writing the article

E - Critical revision of the article

F - Final approval of article

\section{Effectiveness of Neuromuscular exercises (NEMEX) in knee osteoarthritis: A Systematic Review with meta-analysis}

\author{
Jyoti Sabharwal*A-E (D), Shabnam Joshi ${ }^{\mathrm{E}-\mathrm{F}}$ (D), Rekha Chaturvedi ${ }^{\mathrm{B}-\mathrm{C}}$ (D), \\ Meenakshi Bagri ${ }^{\mathrm{B}, \mathrm{E}}$ (D), Vandana Rani ${ }^{\mathrm{B}, \mathrm{E}}$ (iD
}

Guru Jambheshwar University of Science \& Technology, Hisar, India

*Correspondence: Jyoti Sabharwal, Guru Jambheshwar University of Science \& Technology, Hisar; email: jyotisabharwal35@gmail.com

\begin{abstract}
The main objective of the article was to evaluate the effectiveness of Neuromuscular exercises (NEMEX) on pain and function in patients with knee osteoarthritis. Pubmed, Cochrane, PEDro and Google Scholar were searched. The eligibility criteria were: Randomized controlled trials, single blinded controlled trials, controlled before and after study comparing and assessing the effectiveness of NEMEX in knee osteoarthritis, articles published in English language till 2020 with NEMEX either alone or in conjunction with other interventions (drugs, educational packages) in patients with knee osteoarthritis. Outcome measures used for meta-analysis were visual analog scale (VAS) for pain and Western Ontario and McMaster Universities Osteoarthritis Index (WOMAC) for function and the other measures Knee Injury Osteoarthritis Outcome Score (KOOS), Knee Index and Knee Adduction Moment (KAM) and Hip Disability and Osteoarthritis Outcome Score (HOOS). The result of the meta-analysis for the included two studies showed statistically significant reduction in pain (VAS) in the NEMEX group as compared to the other group $Z=0.64, p=0.03, I 2=79 \%$ and no significant reduction in WOMAC scores $\mathrm{Z}=0.70, \mathrm{P}=0.64$. The study concludes that there was statistically significant improvement in pain (VAS) but no functional improvement (WOMAC) with the NEMEX whether used in isolation or in conjunction with other interventions was seen in the patients with knee OA. However, large numbers of studies are required to generalize the effectiveness of NEMEX in knee osteoarthritis. Based on this review, NEMEX can be used as a potential intervention in reducing pain, improving strength and function in knee osteoarthritis patients.
\end{abstract}

Keywords: osteoarthritis, visual analog scale, range of motion, knee joint, gonarthrosis

\section{Introduction}

Osteoarthritis (OA) is the most common rheumatic disease that primarily affects the articular cartilage and the subchondral bone of a synovial joint that results in pain and increased difficulty with functional activities. There is a largeer contribution of knee OA for walking disability, stair climbing and housekeeping than any other disease [1-3]. Osteoarthritis of the knee joint presents with the symptoms such as pain, swelling, instability and decreased range of motion (ROM). These symptoms lead to functional impairment, disability and difficulty in activities of daily living [4,5]. These disease-related factors can potentially be improved with the exercises $[6,7]$.

The quadriceps muscle act as a shock absorber for the knee joint and the weakness in the quadriceps and

This is an Open Access journal, all articles are distributed under the terms of the Creative Commons Attribution-NonCommercial-ShareAlike 4.0 International (CC BY-NC-SA 4.0). License (http://creativecommons. org/licenses/by-nc-sa/4.0/). 
muscles of thigh alters the joint mechanics, resulting in overload at the knee joint [8]. Various studies have suggested that exercises are the cornerstone in the management of the OA and should be given as first line of treatment to all the patients [9]. There is an analgesic effect of the exercises in OA patients that helps in improving the physical function and quality of life [10]. Patients with OA have impaired sensorimotor function, altered muscle activation patterns and reduced functional performances $[7,8,10,11]$. It seems apparent that training programs addressing various aspects of sensorimotor function to improve function and alleviate symptoms can be beneficial in knee OA patients.

Another commonly encountered problem in OA patients is functional instability and defective neuromuscular function [12]. Therefore, the neuromuscular training must be incorporated in the management of patients with knee OA. Neuromuscular exercises (NEMEX) are individualized and goal-based exercise program that address the functional instability and impaired muscle function for the lower extremities. NEMEX typically involve multiple joints and muscle groups that perform in functional weight-bearing positions. Neuromuscular exercises specifically target neuromuscular impairments in patients with knee osteoarthritis. The major goal of the NEMEX is to improve the functional joint stabilization, neuromuscular control and strength. The neuromuscular training is based on biomechanical and neuromuscular principles [13].The principles are based on active movements that are performed in synergy along with initiating the normal movement to achieve the bilateral motor learning effect. NEMEX consists of three phases: warming up phase, NEMEX and cooling down phase. Warm up phase includes the general ergo meter cycle, treadmill walking for about 10 minutes [14]. Second phase consist of posture control, strength of lower extremity, balance, and functional stabilization of trunk and knee. And the cooling down phase includes gait retraining, backward walking (10 minutes) $[15,16]$. These exercises are performed in closed kinetic chain for even distribution of pressure on the articular surface. Muscular co-activation and quality of performance is also an important aspect to be followed in neuromuscular exercises. These exercises influence the knee joint loading and also may be beneficial in patients with varus thrust. There is direct relationship between pain and varus thrust and neuromuscular exercises alters that varus thrust and ultimately pain get reduced.

The effect of neuromuscular exercises has been explored in knee injuries and other injuries of the lower extremities $[17,18]$. However, its use is not well explored in musculoskeletal conditions such as knee OA. Various studies have targeted the effectiveness of different muscle strengthening exercise programs in knee OA patients but, have produced conflicting results making it difficult to recommend specific exercise for the patients with knee OA. A very few studies have explored the effects of unique exercise such as neuromuscular exercises which specifically target functional joint stabilization and sensorimotor deficits in patients with knee osteoarthritis. Therefore, the present study intends to gather the relevant literature that explores the effect of neuromuscular exercises in knee OA patients.

\section{Materials and methods}

\section{Eligibility criteria}

\section{Inclusion criteria}

This systematic review was done according to PRISMA guidelines. This study was registered at the PROSPERO 2020 CRD42020173499. All English language, full text, randomized controlled trials, single blinded controlled trial and controlled before and after study from year 2010 to 2020 assessing the efficacy of neuromuscular exercises in knee osteoarthritis with outcome variables visual analog scale (VAS), Western Ontario and McMaster Universities Osteoarthritis Index (WOMAC), Knee Injury Osteoarthritis Outcome Score (KOOS), Knee Index and Knee Adduction Moment (KAM) and Hip Disability and Osteoarthritis Outcome Score (HOOS) were included for the systematic review and VAS, WOMAC for meta - analysis.

\section{Exclusion criteria}

Articles in other language, full text unavailable, not randomized controlled trials were excluded for this review.

\section{Information sources}

Pubmed, Cochrane, PEDro databases and Google Scholar were used as the source for locating the literature. The search was done in last week of February 2020. A supplementary electronic search was done to update the original search in February 2021 that included all the above databases.

\section{Search strategy}

To search the literature in Pubmed, keywords in the advanced search option used were: "Neuromuscular exercises" OR "NEMEX" AND "Osteoarthritis" OR "OA" AND "knee" all in Title/Abstract. To scrutinize the articles, Filters used were: Randomized Controlled Trial and Humans. For searching the literature in Cochrane, keywords used were: ("Neuromuscular exercises"): ti, ab, kw OR ("NEMEX"): ti, ab, kw AND ("Osteoarthritis"): ti, ab, kw OR ("OA"). For searching in Pedro, 
keywords used were: ("Neuromuscular exercises"): ti, ab, kw AND ("knee Osteoarthritis"): ti, ab, kw.

\section{Study selection}

Eligible and relevant studies in which NEMEX was given in isolation or in combination with other interventions (drugs, educational packages) to the patients of knee OA were selected by title first and by title and abstract then and lastly by the availability of the full text by one reviewer (JS). Studies that included VAS for pain, WOMAC for function, KOOS, KAM and HOOS were considered for this review. Studies were excluded from this systematic review if data in terms of mean and standard deviation (SD) were unavailable.

\section{Data collection}

The search was done independently by two authors (SJ, RC) and data was drawn out according to the MeSH term and related keywords. Any conflict was fixed after conversation with another author (SJ) and the majority option was chosen.

\section{Data extraction}

To determine the efficacy of the intervention, mean and standard deviation (SD) of pain (VAS) and function (WOMAC) from both the experimental and control group were extracted. Data for author/year, country/ continent and total number of subjects for both the groups were also extracted and compared.

\section{Risk of bias in individual studies}

PEDro score was used to assess the methodological study quality. The studies meeting the criteria for randomization and allocation concealment, assessor blinding and intention-to-treat analysis were scored with more than 7 scoring in PEDro. Quality assessment was done by two investigators (JS, SJ) independently.

\section{Synthesis of results}

Results were synthesized using Review Manager 5 (RevMan 5.3), a Cochrane Collaboration's software for systemic reviews and meta-analysis. The data was entered for mean, SD and total number of the subjects for both NEMEX group and the other group and mean difference and $95 \% \mathrm{CI}$ were calculated. The level of Significance was 0.05 and the analysis was done by two independent investigators (JS, SJ).

\section{Results}

The initial yield was 85 papers from the primary search. From these, 24 duplicates were discarded and on the basis of the title and abstract, 22 references were excluded. There were 3 study protocols, 9 registered clinical trials and 15 others were also excluded. And out of the 85 papers 15 full text articles (RCTs) were assessed for the eligibility. 8 articles out of the 15 articles were excluded which don't comply the selection criteria. All seven studies which met the inclusion criteria were randomized clinical trials $[19-24,25]$ that were included for the systematic review and out of 7 studies 5 were excluded because the desired outcome was not reported. And the remaining 2 studies [19,20] were used for meta-analysis. In the supplementary search, no paper fulfill the inclusion criteria and subsequently no further studies were incorporated in the review. Figure 1 depicts the PRISMA flow diagram.

\section{Study characteristics}

All the studies included in this review were randomized controlled trials having 765 patients in total. Three studies were from Australia [19-21] three from Denmark [22-24] and in the other study participants were recruited from Sweden [25]. All the studies used NEMEX either alone or in combination with other intervention and were compared to the other group. Two studies compared the NEMEX with quadriceps strengthening (QS) $[19,20]$. One study used 3-month treatment program with MEDIC group compared to usual care in patients with knee osteoarthritis [21]. One study assessed the patients with medial knee OA enrolled in a 12-week treatment program, comparing neuromuscular exercise to quadriceps strengthening exercises [22]. One study compared NEMEX group and Pharma group [23]. One used 8 week of neuromuscular exercises programme [24]. One study included patients of both hip and knee osteoarthritis [25]. All the studies had measured changes in pain (VAS) and functional assessment: WOMAC, KOOS, KAM, HOOS. Table 1 summarizes the findings of the included studies.

The result of meta-analysis suggests that there was a significant reduction in pain (VAS) with $\mathrm{df}=1, \mathrm{P}=$ $0.03, \mathrm{Z}=0.64(0.52)$ and $\mathrm{I}^{2}=79 \%$. But there is no significant functional improvement with $\mathrm{df}=1, \mathrm{P}=0.64$, $\mathrm{Z}=0.70$ and $\mathrm{I}^{2}=0 \%$. Figure 2 present forest plot for pain (VAS) in patients with knee Osteoarthritis and figure 3 funnel plot for pain (VAS) in patients with knee Osteoarthritis.

\section{Quality assessment}

Table 2 summarizes the quality of studies included in this review. All the included studies have PEDRO score of above 7 out of 11 that shows high quality of the studies. Table 3 summarizes risk of bias by Cochrane collaboration modified tool. 


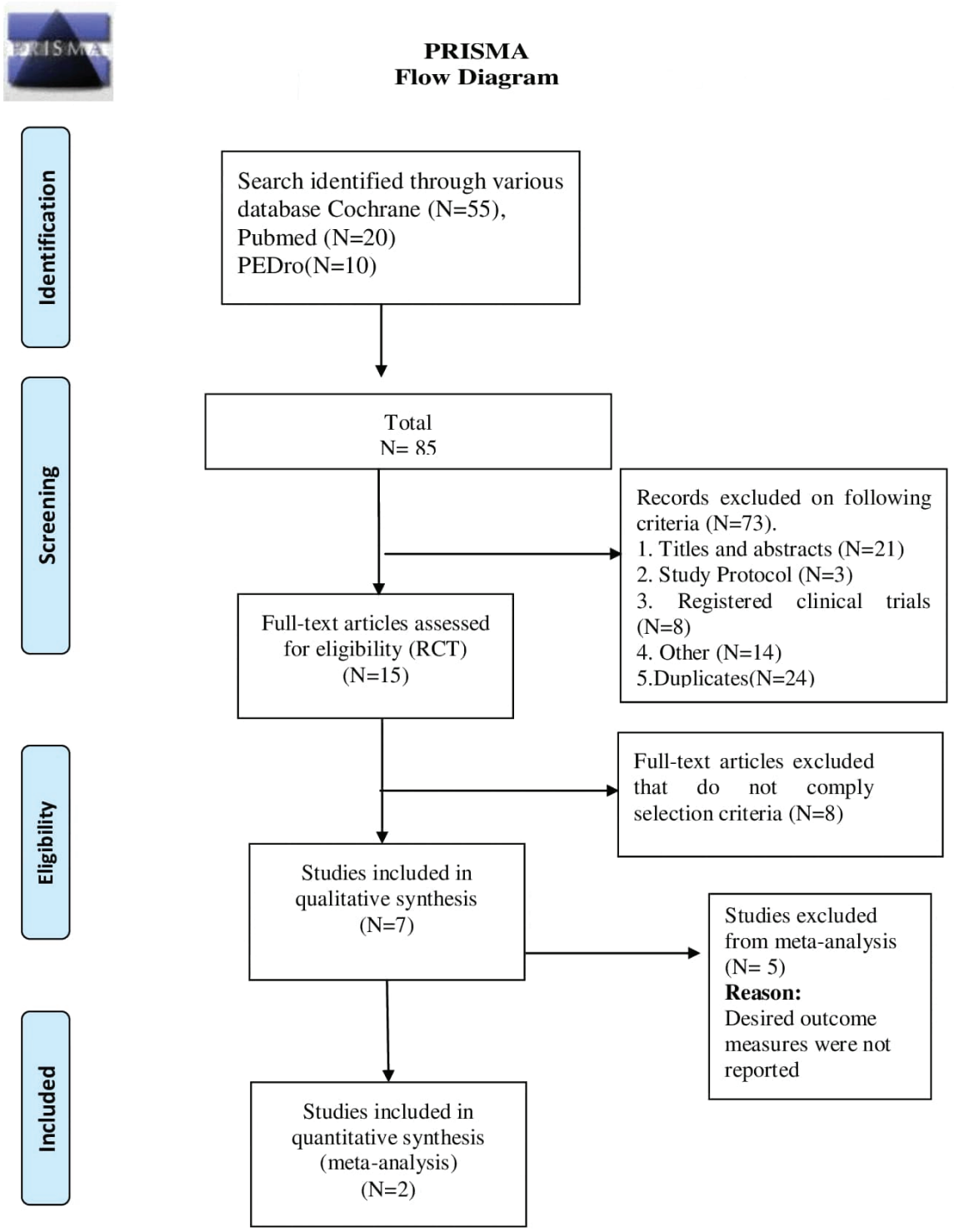

Fig. 1. PRISMA flow diagram

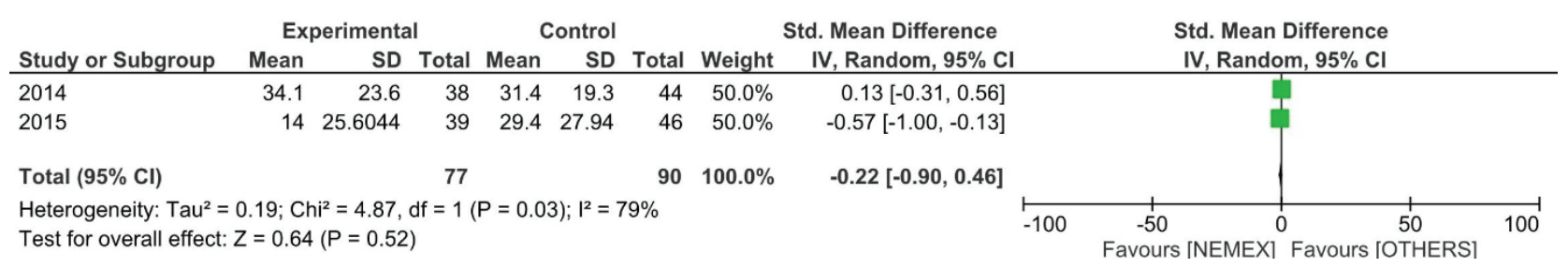

Fig. 2. Forest plot for pain (VAS) in patients with knee osteoarthritis

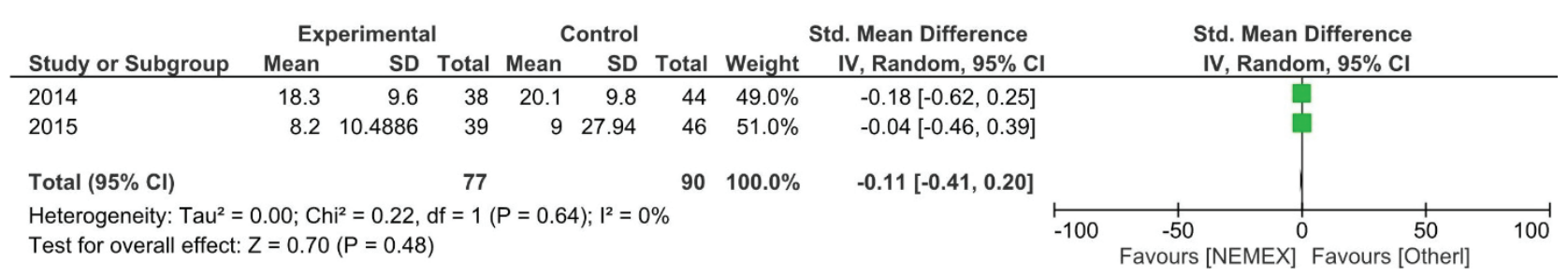

Fig. 3. Forest plot for function (WOMAC) in patients with knee osteoarthritis 


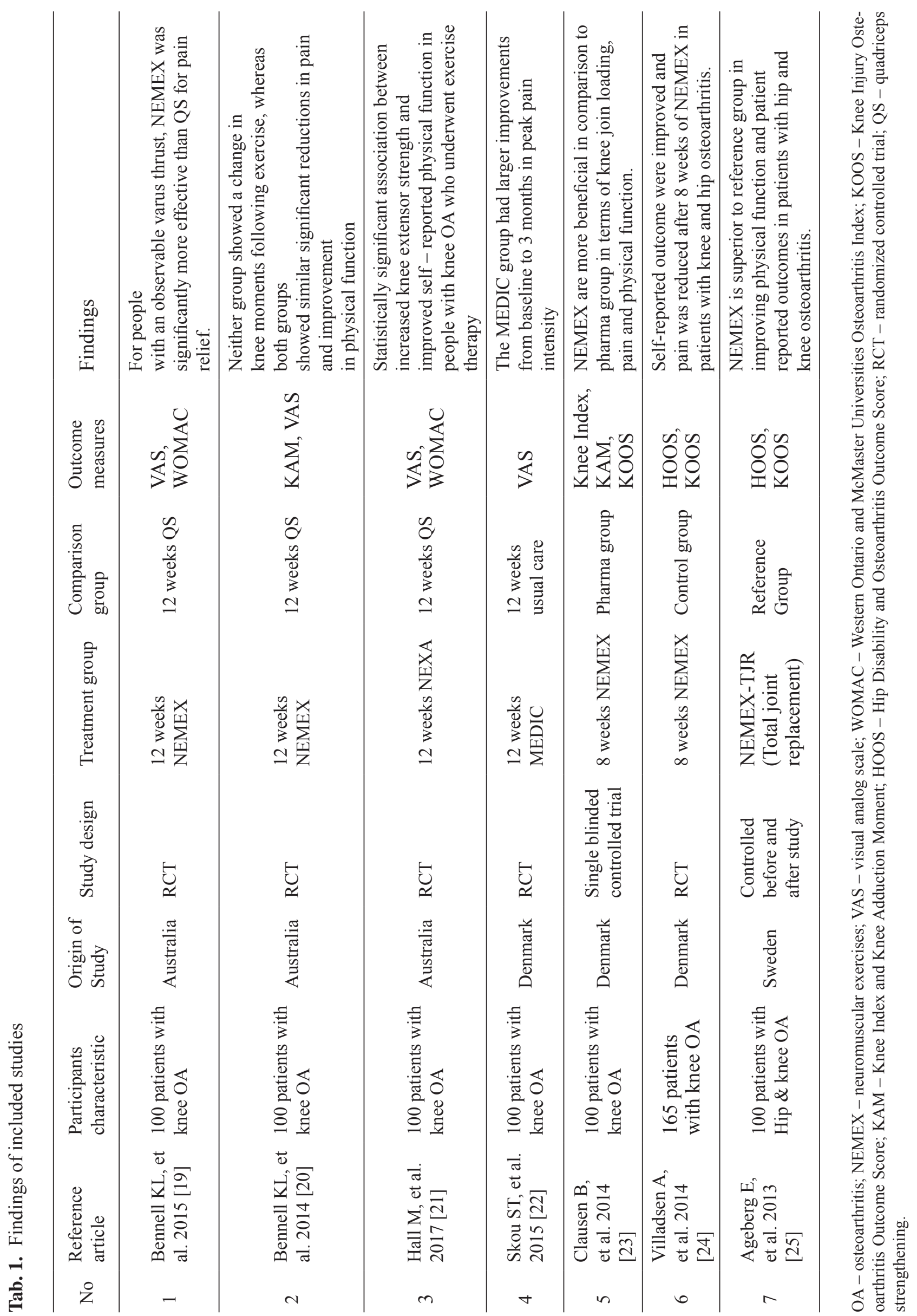


Tab. 2. Quality assessment of selected Randomized Controlled Trials using Physiotherapy Evidence Database (PEDro scoring)

\begin{tabular}{|c|c|c|c|c|c|c|c|c|}
\hline S.No. & & $\begin{array}{l}\text { Bennell KL, } \\
\text { et al. 2015 } \\
\text { [19] }\end{array}$ & $\begin{array}{l}\text { Bennell KL, } \\
\text { et al. 2014 } \\
{[20]}\end{array}$ & $\begin{array}{l}\text { Skou ST, } \\
\text { et al. 2015 } \\
{[21]}\end{array}$ & $\begin{array}{c}\text { Hall, et al. } \\
2017 \\
{[22]}\end{array}$ & $\begin{array}{c}\text { Clausen B, } \\
\text { et al. 2014 } \\
\text { [23] }\end{array}$ & $\begin{array}{c}\text { Villadsen A, } \\
\text { et al. 2014 } \\
\text { [24] }\end{array}$ & $\begin{array}{c}\text { Ageberg E, } \\
\text { at al. 2013 } \\
{[25]}\end{array}$ \\
\hline 1 & $\begin{array}{l}\text { Specified eligibility } \\
\text { criteria }\end{array}$ & Yes & Yes & Yes & Yes & Yes & Yes & Yes \\
\hline 2 & Random allocation & Yes & Yes & Yes & Yes & Yes & Yes & Yes \\
\hline 3 & Concealed allocation & No & Yes & Yes & Yes & Yes & Yes & Yes \\
\hline 4 & Similar baseline & Yes & Yes & Yes & Yes & Yes & Yes & Yes \\
\hline 5 & Subjects blinding & No & No & No & No & No & No & No \\
\hline 6 & Therapists blinding & No & No & No & No & No & No & No \\
\hline 7 & Assessors blinding & Yes & Yes & Yes & Yes & No & Yes & No \\
\hline 8 & $\begin{array}{l}\text { Measures of key } \\
\text { outcomes from more } \\
\text { than } 85 \% \text { of subjects }\end{array}$ & No & No & Yes & No & No & Yes & Yes \\
\hline 9 & $\begin{array}{l}\text { Intention to treat } \\
\text { analysis of one key } \\
\text { outcome }\end{array}$ & Yes & Yes & Yes & Yes & Yes & Yes & No \\
\hline 10 & $\begin{array}{l}\text { Statistical comparisons } \\
\text { between-group of at } \\
\text { least one key outcome }\end{array}$ & Yes & Yes & Yes & Yes & Yes & Yes & Yes \\
\hline 11 & $\begin{array}{l}\text { Variability for at least } \\
\text { one key outcome }\end{array}$ & Yes & Yes & Yes & Yes & Yes & Yes & Yes \\
\hline Total & & $7 / 11$ & $8 / 11$ & $9 / 11$ & $8 / 11$ & $7 / 11$ & $9 / 11$ & $7 / 11$ \\
\hline
\end{tabular}

Tab. 3. Risk of Bias by Cochrane collaboration modified tool

\begin{tabular}{lccccccc}
\hline & $\begin{array}{c}\text { Bennell KL, } \\
\text { et al. 2015 } \\
{[19]}\end{array}$ & $\begin{array}{c}\text { Bennell KL, } \\
\text { et al. 2014 } \\
{[20]}\end{array}$ & $\begin{array}{c}\text { Skou ST, } \\
\text { et al. 2015 } \\
{[21]}\end{array}$ & $\begin{array}{c}\text { Hall M, } \\
\text { et al. 2017 } \\
{[22]}\end{array}$ & $\begin{array}{c}\text { Clausen B, } \\
\text { et al. 2014 } \\
{[23]}\end{array}$ & $\begin{array}{c}\text { Villadsen A, } \\
\text { et al. 2014 } \\
{[24]}\end{array}$ & $\begin{array}{c}\text { Ageberg E, } \\
\text { et al. 2013 } \\
{[25]}\end{array}$ \\
\hline $\begin{array}{l}\text { 1. Random sequence } \\
\text { generation (Selection bias) }\end{array}$ & Low Risk & Low Risk & Low Risk & Low Risk & Low Risk & Low Risk & Low Risk \\
$\begin{array}{l}\text { 2.Allocation concealment } \\
\text { (Selection bias) }\end{array}$ & High Risk & Low Risk & Low Risk & Low Risk & Low Risk & Low Risk & Low Risk \\
$\begin{array}{l}\text { 3.Blinding of participants } \\
\text { and personnel (Performance } \\
\text { bias) }\end{array}$ & High Risk & High Risk & High Risk & High Risk & High Risk & High Risk & High Risk \\
$\begin{array}{l}\text { 4. Blinding of outcome } \\
\text { assessment (Detection bias) }\end{array}$ & Low Risk & Low Risk & Low Risk & Low Risk & Low Risk & Low Risk & Low Risk \\
$\begin{array}{l}\text { 5.Incomplete outcome } \\
\text { data(Attrition bias) }\end{array}$ & Low Risk & Low Risk & Low Risk & Low Risk & Low Risk & Low Risk & Low Risk \\
$\begin{array}{l}\text { 6.Selective reporting } \\
\begin{array}{l}\text { (Reporting bias) } \\
\text { 7. Other bias }\end{array}\end{array}$ & Low Risk & Low Risk & Low Risk & Low Risk & Low Risk & Low Risk & Low Risk \\
\hline
\end{tabular}




\section{Discussion}

The aim of the study was to assess the effectiveness of NEMEX in patients with knee OA. The neuromuscular exercises are based on biomechanical and neuromuscular principles and aims to improve sensorimotor control and achieve functional stability. Strengthening exercises are used for people with OA but the neuromuscular exercises were developed to target the sensorimotor deficiencies and functional instability associated with knee injuries as well as knee osteoarthritis. By addressing the quality of movement in all 3 movement planes, NEMEX improve the sensorimotor control and also assist in attaining functional joint stabilization. Various studies have found that NEMEX are effective in relieving pain alters knee biomechanics, improves the function and muscle-activation patterns of the knee in patients with knee OA and also in sports injuries like ligament and meniscal tears. Neuromuscular training also plays an important role in preventing the ligament injuries in male soccer players. Furthermore, NEMEX has been shown to improve articular cartilage quality in middle-aged patients who have had meniscectomies and are at high risk of development of Knee OA. Therefore the NEMEX play an important role in the management of knee OA.

Neuromuscular exercises also helpful in improving the muscular strength and performance of the patients. In comparison with other exercise protocols neuromuscular exercises mainly improves the balance, coordination as well as quality of movement which are the key factors in maintaining the joint stability. The studies included in this review varied widely in terms of population, group's comparison, types and durations of the intervention. Overall, the included studies had high methodological qualities with an average score of 7 on the PEDro scale. One study found significant improvements in pain and physical function in both groups (NEMEX group and quadriceps group). Neuromuscular exercises was more effective in patients of knee OA with varus thrust as the varus thrust is directly related with pain and neuromuscular exercises alter that thrust which decreases the pain but no significant improvement in KAM which is the main factor in genesis of knee osteoarthritis [19,20]. Previous study in 2015 compared the MEDIC group which included 3 months protocol of exercises, education, diet, insoles and medications with usual care group and in that, MEDIC group showed larger improvements in peak pain intensity $(\mathrm{p}=0.02)$ from baseline to 3 months and function on long term basis [21].

Various research evidences have suggested that NEMEX is effective in other injuries and disorders other than knee osteoarthritis like ligament injuries, childhood obesity, balance and functional improvement $[13,17]$. It was also found that 8 weeks of neuromuscular exercises is more beneficial than use of medications (analgesic, anti -inflammatory drugs) in terms of knee joint loading, knee pain and physical function [23]. Neuromuscular exercises are feasible in patients with knee osteoarthritis and pain was relieved and performance of patients was improved in patients with knee and hip osteoarthritis [24].

This systematic review included only two studies for the meta-analysis and the included studies had high heterogeneity, making it difficult to conclude the effectiveness of NEMEX in knee osteoarthritis. The result of meta-analysis suggested that statistically significant improvement was there in the pain (VAS) but not for functional improvement (WOMAC). In order to generalize the efficacy of NEMEX, more studies with large sample size are required to determine the role of NEMEX in the treatment of knee osteoarthritis. Taking in the account the promising results shown by the NEMEX in various conditions and to some extent in knee OA, its efficacy can be further explored and may prove to be a potential intervention in management of knee osteoarthritis.

\section{Limitations}

There were variations in combination of NEMEX with quadriceps strengthening, usual care and medic care for treatment of osteoarthritis that might have caused bias in the analysis. Large sample size is required to generalize the effectiveness of NEMEX.

\section{Conclusion}

The result of the meta-analysis suggests that there is a statistically significant reduction in pain with in patients with knee OA after NEMEX. The neuromuscular exercises can be the choice of intervention for reducing pain in patients with knee osteoarthritis. The effectiveness of NEMEX must be explored further but it can be recommended in patients with knee osteoarthritis for better management of pain.

\section{Funding}

This research received no external funding.

\section{Conflict of interests}

The authors have no conflict of interest to declare.

\section{References}

1. Davis MA, Ettinger WH, Neuhaus JM, et al. Knee osteoarthritis and physical functioning: evidence from the NHANES 1 epidemiologic follow-up study. J Rheumatol. 1991; 18(4): 591-8. 
2. Guccione AA, Felson DT, Anderson JJ, et al. The effects of specific medical conditions on the functional limitations of elders in the Framingham study. Am J Public Health. 1994; 84(3): 351-8.

3. Van Dijk GM, Dekker J, Vennhof C, et al. Course of functional status and pain in osteoarthritis of the hip or knee: a systematic review of the literature. Arthritis Rheum. 2006; 55(5): 779-85.

4. Maurer BT, Stern AG, Kinossian B, Cook KD, Schumacher HR. Osteoarthritis of the knee: isokinetic quadriceps exercise versus and educational intervention. Arch Phys Med Rehabil. 1999; 80(10): 1293-99.

5. Roos EM, Herzog W, Block JA, Bennell KL. Muscle weakness, afferent sensory dysfunction and exercise in knee osteoarthritis. Nat Rev Rheumatol. 2011; 7(1): 57-63.

6. Buchner DM, Beresford SAA, Larson EB, et al. Effects of physical activity on health status in older adults II: Intervention studies. Ann Rev Publ Health. 1992; 13(1): 469-88.

7. Fiatarone MA, Evans WJ. The etiology and reversibility of muscle dysfunction in the aged. J Gerontol. 1993; 48: 77-83.

8. Knoop J, Steultjens MP, van der Leeden M, van der Esch M, Thorstensson CA, Roorda LD, et al. Proprioception in knee osteoarthritis: a narrative review. Osteoarth Cartil. 2011; 19(4): 381-8.

9. Zhang W, Moskowitz RW, Nuki G, Abramson S, Altman RD, Arden N, et al. OARSI recommendations for the management of hip and knee osteoarthritis, Part II: OARSI evidence-based, expert consensus guidelines. Osteoarth Cartil. 2008; 16(2): 137-62.

10. Bennell KL, Hunt MA, Wrigley TV, Lim BW, Hinman RS. Role of muscle in the genesis and management of knee osteoarthritis. Rheum Dis Clin North Am. 2008; 34(3): 731-54.

11. Dekker J, van Dijk GM, Veenhof C. Risk factors for functional decline in osteoarthritis of the hip or knee. Curr Opin Rheumatol. 2009; 21(5): 520-4.

12. Ageberg E. Consequences of a ligament injury on neuromuscular function and relevance to rehabilitation-using the anterior cruciate ligament-injured knee as model. J Electromyogr Kinesiol. 2002; 12(3): 205-12.

13. Zatterstrom R, Friden T, Lindstrand A, Moritz U. Muscle training in chronic anterior cruciate ligament insufficiency-a comparative study. Scand J Rehabil Med. 1992; 24(2): 91-7.

14. Borg G, Ljunggren G, Ceci R. The increase of perceived exertion, aches and pain in the legs, heart rate and blood lactate during exercise on a bicycle ergometer. Eur J Appl Physiol Occup Physiol. 1985; 54(4): 343-9.

15. Ageberg E, Link A, Roos EM. Feasibility of neuromuscular training in patients with severe hip or knee OA: the individualized goal-based NEMEX-TJR training program. BMC Musculoskelet Disord. 2010; 11: 126.
16. Villadsen A, Overgaard S, Holsgaard-Larsen A, Christensen R, Roos EM. Postoperative effects of neuromuscular exercise prior to hip or knee arthroplasty: a randomised controlled trial. Ann Rheum Dis. 2014; 73(6): 1130-7.

17. Zatterstrom R, Friden T, Lindstrand A, Moritz U. Early rehabilitation of acute anterior cruciate ligament injury-a randomized clinical trial. Scand J Med Sci Sports. 1998; 8(3): 154-9.

18. Ageberg E, Zatterstrom R, Moritz U, Friden T. Influence of supervised and nonsupervised training on postural control after an acute anterior cruciate ligament rupture: A 3-year longitudinal prospective study. J Orthop Sports Phys Ther. 2001; 31(11): 632-44.

19. Bennell KL, Dobson F, Roos EM, Skou ST, Hodges P, Wrigley TV, et al. Influence of Biomechanical Characteristics on Pain and Function Outcomes From Exercise in Medial Knee Osteoarthritis and Varus Malalignment: Exploratory Analyses From a Randomized Controlled Trial. Arthritis Care Res. 2015; 67(9): 1281-8.

20. Bennell KL, Kyriakides M, Metcalf B, Egerton T, Wrigley TV, Hodges PW, et al. Neuromuscular Versus Quadriceps Strengthening Exercise in Patients With Medial Knee Osteoarthritis and Varus Malalignment. Arthritis Rheumatol. 2014; 66(10): 950-9.

21. Hall M, Hinman RS, van der Esch M, van der Leeden M, Kasza J, Wrigley TV, et al. Is the relationship between increased knee muscle strength and improved physical function following exercise dependent on baseline physical function status? Arthritis Res Ther. 2017; 19(1): 1-9.

22. Skou ST, Roos EM, Simonsen O, Laursen MB, Rathleff MS, Arendt-Nielsen L, et al. The efficacy of nonsurgical treatment on pain and sensitization in patients with knee osteoarthritis: a pre-defined ancillary analysis from a randomized controlled trial. Osteoarthritis Cartilage. 2015; 24(1): 108-16.

23. Clausen B, Holsgaard-Larsen A, Søndergaard J, Christensen R, Andriacchi TP, Roos EM. The effect on knee-joint load of instruction in analgesic use compared with neuromuscular exercise in patients with knee osteoarthritis: study protocol for a randomized, single-blind, controlled trial (the EXERPHARMA trial). Trials. 2014; 15(1): 1-14.

24. Villadsen A, Overgaard S, Holsgaard-Larsen A, Christensen R, Roos EM. Immediate efficacy of neuromuscular exercise in patients with severe osteoarthritis of the hip or knee: a secondary analysis from a randomized controlled trial. J Rheumatol. 2014 ; 41(7): 1385-94.

25. Ageberg E, Nilsdotter A, Kosek E, Roos EM. Effects of neuromuscular training (NEMEX-TJR) on patient-reported outcomes and physical function in severe primary hip or knee osteoarthritis: a controlled before-and-after study. BMC Musculoskelet Disord. 2013; 14(1): 1-4. 